\title{
Italique
}

Poésie italienne de la Renaissance

XIX | 2016

Varia

\section{Tra lirica e epistolografia. Appunti sull'opera poetica di Lodovico Dolce}

Paolo Marini

\section{CpenEdition}

Journals

Edizione digitale

URL: http://journals.openedition.org/italique/444

DOI: $10.4000 /$ italique.444

ISSN: 1663-4438

\section{Editore}

Librairie Droz

\section{Edizione cartacea}

Data di pubblicazione: 1 dicembre 2016

Paginazione: 113-130

ISBN: 978-2-600-04777-7

ISSN: 1423-3983

Notizia bibliografica digitale

Paolo Marini, «Tra lirica e epistolografia. Appunti sull'opera poetica di Lodovico Dolce », Italique [Online], XIX | 2016, online dal 01 décembre 2018, consultato il 07 septembre 2019. URL : http:// journals.openedition.org/italique/444; DOI : 10.4000/italique.444 


$$
\text { PAOLO MAR I N I }
$$

TRA LIRICA E E PISTOLOGR A F A. A P P U N T S ULL'OPERA POETICA D I L O D V I C O D O L C E 



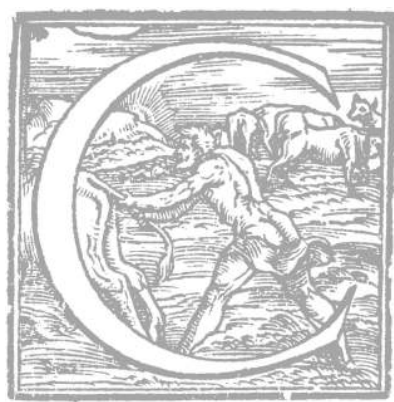

on queste parole, in data $I^{\circ}$ agosto 1538 , Giacomo Marmitta introduce il primo frammento noto della corrispondenza intrattenuta con Lodovico Dolce:

Magnifico Messer Lodovico mio dolcissimo, ecco ch'io vengo a gli inchiostri, eccomi all'epistole, o potreste dire c'ho rubato questo, ecco, all'Aretino, patientia, eccomi dico dalla poesia tratto all'epistole, ma di che genere non so io, o grave, o giocoso, o famigliare. ${ }^{\mathrm{I}}$

Il passaggio dal medium poetico a quello epistolare è osservato come sbocco naturale nell'evoluzione del dialogo fra due letterati di pieno Rinascimento. Uno slittamento, per attrazione ma senza attriti, fra generi istintivamente percepiti come contigui che, con la leggerezza propria del contesto confidenziale, viene marcato evocando il nume tutelare dell'Aretino, autore da pochi mesi del primo libro di lettere in volgare. Non sorprende, dunque, che al centro di composizioni epistolari cosi concepite resti sempre lo spazio condiviso della lirica, evocata esplicitamente col rinvio a un testo o attraverso la menzione di altri sodali che prendono variamente parte al gioco di corrispondenze di cui si sostanzia la societas del petrarchismo cinquecentesco. Così, nella chiusa della lettera, si torna alla poesia con la topica lamentatio di Marmitta sulla lontananza dall'amico risolta in una richiesta di invio di nuovi prodotti poetici e di una non precisata elegia:

Ma lasciando da parte le belle parole, le qual tutte al parer mio sono argumento di poca fede verso l'amico, voi potrete adunque mal grado suo raddolcire questa cotanta amarezza, e so che 'l farete per vostra innata cortesia, co' dolci frutti del vostro alto intelletto, e primeramente per arra di ciò vi degnerete di mandarmi la mia Elegia, che pur mia è, prima che giunga un'altra luna d'Aprile. ${ }^{2}$

Segue un poscritto che, oltre alla richiesta di una copia dell'edizione marcoliniana delle Prose bembesche, contiene «un laco di saluti» ai comuni amici veneziani: tra questi anche Paolo Crivelli, figura sensibile alle istanze filoriformate che proprio a Marmitta dedicherà un sonetto incluso nell'edizione del Is46 della prima antologia giolitina. ${ }^{3}$ 
A tal punto istituzionalizzato il traffico di versi per via epistolare, che nella successiva lettera del I ottobre I538 Marmitta può permettersi di sceneggiare la pratica stessa del mutuo scambio stabilito con Dolce. Nei toni di un garbato understatement rivolto all'esignità della propria vena artistica ("un sonettazzo», "qualche versazzi») il gentiluomo parmigiano gioca a polemizzare sull'incongruo do ut des in atto col piu prolifico collega:

Egli si par bene che voi non pensaste molto a chi di noi duo tenesse più di quel del compagno, quando vi usciro di mano quelle parole: «Oimé ditemi, non tenete voi per ogni verso che io tengo de' vostri dieci de' miei?». Sì, tenete voi. [...] Or via mandimisi la mia Elegia, e delle altre cose vostre se mi amate, e più non si disputi fra noi, ch'io farò il simigliante, se mi verranno fatti qualche versazzi anch'io, più risguardo havendo al desiderio vostro che all'honor mio. ${ }^{4}$

Come è evidente anche da queste poche righe - peraltro rappresentative di un uso ben radicato nel panorama letterario del tempo -, non si tratta solo di un banale espediente per agevolare la circolazione di testi poetici alla spicciolata per vie alternative rispetto ai più canonici percorsi della tradizione. Al contrario, lo spazio epistolare diventa una cassa di risonanza perfettamente funzionale all' amplificazione di vari aspetti di un fenomeno culturale complesso come quello del petrarchismo: dalla discussione di questioni di poetica; alla promozione del profilo artistico di un collega che si intende accreditare in seno alla res publica litterarum; alla semplice commissione di un sonetto d'occasione; al racconto di una fruizione pubblica della poesia, non di rado proposta in contesto accademico; alla richiesta di expertise sulla qualità di un componimento; fino alle varie «forme di commento diffuso» di testi antichi e contemporanei osservate da Franco Tomasi. 'L'interazione fra $i$ due generi passepartout consolida cosi un arengo letterario che si fonda sulla comune matrice dialogica, costitutiva nel caso della forma epistolare e acquisita progressivamente nel caso della forma poetica, dalla tenzone duecentesca al fenomeno - esploso in dimensioni senza precedenti nel corso del Cinquecento - delle rime di corrispondenza.

Inutile dire che nello specifico di una figura di mediazione come quella di Dolce, coinvolta tra l'altro in vesti di allestitore nell'assemblaggio della serie antologica inangurata da Giolito, queste dinamiche risaltano con ancora maggior rilievo. È quanto si ricava dall'incrocio dei dati 
emersi dallo spoglio dell'epistolario con le prime notizie provenienti dal progetto di edizione critica della lirica, un cantiere che ha sin qui condotto all'acquisizione di circa 170 testi, in massima parte sonetti. ${ }^{7}$ Un primo elemento significativo è dato dalla presenza capillare della lirica tra le lettere scritte e ricevute da Dolce. Anche limitandosi alle corrispondenze principali, colpisce la preponderanza delle missive in cui la poesia è tirata in ballo in forma più o meno diretta, sia come oggetto di scambio che come tema di dibattito: 17 occasioni su 28 con Aretino, 7 su Iz con Varchi, 6 su 7 con Marmitta, 4 su 6 con Crivelli, 2 su 3 con Bembo. A conferma del fatto che, per quanto il profilo critico attualmente consolidato a seguito degli approfondimenti condotti da piu parti negli ultimi 30 anni sia principalmente quello dell'homo typographicus, correttore e traduttore, ma anche grammatico e trattatista, Dolce si affaccia sul proscenio letterario della Serenissima come poeta e tale continua a considerarsi e ad essere considerato nel corso degli anni, senza che questo, ovviamente, escluda le altre, molteplici, competenze. Anzi, si potrebbe dire che proprio sulla riconosciuta familiarità e sulla frequentazione costante con la materia poetica si fondano le sue credenziali di mediatore agli occhi dei colleghi letterati, dell'impresa di Giolito e dello stesso mercato editoriale, destinatario ultimo della sua attività professionale.

Una breve rassegna di alcune delle testimonianze più pregnanti non può che muovere dal carteggio fra Dolce e Aretino e dall'immagine pubblica che il maestro vuole precocemente cucire addosso al suo delfino sin dal primo libro delle Lettere. Gli intenti di messer Pietro sono molto chiari già nella lettera a Veronica Gambara del 2 novembre I536, quando Dolce ha al suo attivo soltanto la prova da petrarchista in erba del Sogno di Parnaso con alcune altre rime d'amore, la traduzione della Poetica oraziana in endecasillabi sciolti (con importante dedica allo stesso Aretino), le ottave del Sacripante, le Stanze per l'impresa di Carlo $V$ a Tunisi e poco altro. Messo in mezzo come corriere in un passaggio di missive tra la contessa di Correggio e Bembo, Aretino si permette di inserire nello scambio una lettera di «M. Lodovico Dolce, a cui forse per merito de le sue nuove vertù non si disdirebbe d'entrar terzo fra voì. Ed è nei panni di compiaciuto cerimoniere di questo rito di patronage che poche righe dopo arriva addirittura ad accostare Dolce «giovane figliuolo de le Muse» al «vecchio padre» Bembo. ${ }^{8}$ Tutt'altro che una boutade episodica: si tratta, al contrario di un passaggio 
fondamentale in una strategia promozionale che si articola in più momenti. Cosi nella lettera alla Gambara del I 8 maggio I 37 la vetrina delle Lettere viene sfruttata per dare pubblicità al sonetto di lode offerto dalla poetessa all'intraprendente neofita, «il quale per sentirsi lodare da colei che dà lo spirito a la laude, è divenuto geloso di se stesso, conoscendo quel che egli è nel mirabile sonetto con cui l'onorate».' La circostanza è illuminata dalla missiva della Gambara a Dolce datata 28 aprile I537:

So che V.S. mi deve aver tenuta meritamente discortese, essendo stata tarda a dar risposta ai due leggiadri Sonetti, e lettera sua. Ma per dirle il vero io restai così fuori di me al primo aspetto di essi, ch'io perdei l'ardire di poter con onor mio soddisfare alla millesima parte dell'obbligo che con V.S. teneva [...]. Ora per voler pur in parte renderle grazie degli obblighi le tengo [...], le scrivo il qui inchiuso Sonetto [...]. Il resto dirà in mia escusazione il divino Signor Pietro, il quale ho pregato voglia, per sua cortesia, supplire a quello, che per più non sapere ho mancato. ${ }^{10}$

Che, al netto delle convenzioni retoriche di cortesia, certifica il gradimento dell'omaggio poetico dolciano da parte della contessa, descrivendo nei fatti uno scambio di rime di corrispondenza, col solito Aretino al centro della partita di giro. Un riconoscimento in altissimis che fa il paio con quello analogo testimoniato dalla lettera di un'altra protagonista della lirica cinquecentesca, Vittoria Colonna, scritta in data Is dicembre I536:

sapendosi che non sono sufficienti le parole a ringratiar l'opera de' vostri divini sonetti, giovarà pure la mia tardanza a discolparmi, perché molti vostri et miei amici vi haveran scritto quanto io gli habbia lodati [...]. Non lassarò perciò di dire che io non apersi mai forsi charta, che mi empisse tanto gli uni et gli altri occhi, come fé la vostra lettera. A quei de la fronte si scoversero minute perle [...]. A quei dell'intelletto si mostrò in un punto Parnaso, Apollo et le Muse nel maggior loro honore haver con somma letitia condito del vostro dolce in modo l'acque d'Helicona, che del suo ambrosia et nettar non han più invidia a Giove. ${ }^{11}$

La sanzione pubblica degli attestati di stima giunti dalle novelle «Safo e Corinna» arriva puntuale sul palcoscenico delle Lettere tramite la missiva di Aretino a Dolce del I7 dicembre I537. Con tanto di invito 
esplicito a tesaurizzare le lettere per esibirle all'occorrenza come credenziali di perfetto letterato:

Eccovi la lettra che vi si scrive Veronica Gambara, non punto differente da quella scrittavi da Vittoria Colonna [...]. Sì che riponetele in luogo che si possin mostrare di tempo in tempo, come gemme de la gloria loro, e come corde del merito de l'istormento del vostro ingegno. ${ }^{\mathrm{I}}$

L'endorsement di Aretino risulta, a ben vedere, tutt'altro che disinteressato. E assodato che in quegli anni Dolce svolge per lui una delicata funzione di pontiere sia verso il contesto accademico padovano che nei confronti di Bembo. Non si spiegherebbe altrimenti la richiesta di un sonetto celebrativo da allegare alle Stanze di Messer Pietro Aretino in lode di Madonna Angela Serena rivolta dallo stesso Dolce all'autore delle Prose con la lettera del I7 novembre I536. ${ }^{\mathrm{I}}$ Ma, soprattutto, Aretino affida alle cure del giovane compare la princeps del secondo libro delle Lettere e la seconda edizione del primo libro, con tanto di investitura ufficiale contenuta nell'epistola del $I^{\circ}$ settembre IS4I, dove Dolce viene esplicitamente definito «nato a comune utilità, come gli uomini buoni». ${ }^{\mathrm{I}}{ }^{4}$ Il dato, di per sé cruciale, va tenuto in considerazione ancora maggiore quando si rilegge un testo fondamentale ai nostri fini come la lettera di Dolce a Aretino datata I8 gingno I537, la XLII della giunta di Lettre diverse a l'autore, introdotta proprio con la nuova edizione del primo volume della silloge aretiniana che esce nell'agosto I542. ${ }^{\mathrm{I}}$

Ora, alla luce del coinvolgimento diretto del letterato veneziano nell'allestimento dell'opera, mi pare si debba osservare la prossimità di questo testo con una delle composizioni più importanti e stilisticamente rappresentative dell'intero primo libro delle Lettere di Aretino, quell'epistola Iss del 25 giugno Is37 che, non a caso, tra le redarioni del I538 e del I542 cambia persino destinatario: da Niccolò Franco allo stesso Dolce. ${ }^{\mathrm{I}}$ La volontà di accostare le due lettere è implicita nella vicinanza delle date distanti solo 7 giorni, coincidenza di sicuro non fortuita nella sostanziale coerenza sistemica - e, dunque, anche cronotopica - su cui si sostiene la fictio letteraria della raccolta. Con ogni probabilità un espediente attraverso il quale si intende suggerire un rapporto dialogico, quasi di botta e risposta, tra testi entrambi centrati su questioni di poetica. Letta in questa prospettiva, come provocatoria sollecitazione del verbo aretiniano, la lettera di Dolce assume uno 
spessore ben diverso. E acquista significato la messa in scena studiatamente petrarchesca, per atmosfere, temi e lessico, della prima parte, dove l'immissione di materiale lirico nel tessuto epistolare è davvero cospicua. Il tutto funzionale al rovesciamento della conclusione, da leggere invece in quella chiave anticlassicistica che prenderà corpo, con ben altra ampiezza, nella lettera di Aretino.

Ma andiamo per ordine. Nell'attacco, un Dolce in preda ai tormenti d'amore - «Amor m'ha concio male», chioserà poco oltre $-{ }^{17}$ si dipinge in un quadretto da letteratura in villa al centro di un locus amoenus ben caratterizzato:

salito in una delle nostre barchette insieme con alcuni gentiluomini miei amici, mi allontanai da questi lidi. E tra lo spazio di poche ore, giù per le onde usate spingendoci il fiato d'un fresco venticello, giunsi al piacevole luogo, dove già alquanti dì sono; che è un piccolo Castello, detto Piove di Sacco $[. .$.$] , molto vago, e molto gentile, sì per la qualità dell'aere dolce \mathrm{e}$ temperato, come del terreno non men fertile che dilettoso. ${ }^{18}$

Su questo sfondo si innesta il tema petrarchesco della fuga nella natura "per alleggiar in qualche parte [...] l'animo dalle gravi infirmità $e$ passioni». Ma, come vuole la topica, la ricerca di un rifugio si dimostra subito illusoria:

Percioché la imagine di quel volto, che io sopra tutte le cose amo, in più verisimile ritratto che di man d'Apelle, o pur del vostro Tiziano, tralucendo nel cuor mio, il quale sempre lo contempla con gli occhi dell'animo, così sdegnoso e pieno d'ira mi si rappresenta, che io non sento canto d'augello alcuno, né veggio dolce verdeggiar d'arboscello, che a me veramente non paia «un diserto, e fiere aspre e selvagge». Il simile m'aviene, se io miro gli tanto dal Petrarca illustrati «fior, frondi, erbe, ombre, antri, onde, aure soavi». ${ }^{19}$

Il tema del ritratto della donna dipinto / scolpito / inciso nel cuore del poeta da Amore, artista senza rivali sia tra gli antichi che tra $i$ moderni, appartiene sin dalle origini al repertorio tradizionale della lirica amorosa. ${ }^{20}$ Dolce lo innesta senza apparenti difficoltà nel corpo della lettera per introdurre una serie di citazioni dirette dal Canzoniere petrarchesco (son. 310 e 303), che sono insieme prelievi di lessico e di oggetti poetici da un modello celebrato ma contestualmente rifiutato nel momento in cui si esalta come unico remedium amoris il «potere 
di quello inchiostro e di quella penna che ha valore d'occider gli nomini, e di tornargli in vita, come aggrada» all'Aretino:

Egli è vero che io ho letto e leggo le più volte i raccordi d'Ovidio, con che lo ingenioso Poeta ne insegna a rompere i legami d'Amore [...]. E so che molto più in ciò mi può giovare una brieve lettera dello Aretino [...]. Per la qual cosa io nel mio male cheggio l'opera vostra [...]. Io mentre questo cerco da voi, Signor Pietro, chi non vede che cerco medesimamente la immortalità? La quale con tale mezzo spero d'avere ne i vostri scritti. ${ }^{21}$

Dove si può leggere sia l'adesione convinta a una poetica del "naturale" che rifugge dal principio di imitazione e si allinea ai principi espressi da Aretino nell'epistola I5); ${ }^{22}$ sia la richiesta di un riconoscimento pubblico da esprimere attraverso il formidabile strumento delle Lettere, cosa che, come si è visto, il venerato maestro non esita a concedere a piene mani in più occasioni.

E precisamente ciò che apprezziamo nella lettera a Dolce del 25 novembre I537, la 249 del primo libro, nella quale Aretino inscena un reciproco omaggio poetico allegando un sonetto di encomio per il compare:

Io, compar, vi scrivo i versi sottoscritti, acciò che non crediate ch'io fugga l'obligo nel quale m'hanno posto i Sonetti con che mi loda l'umanità vostra, e non per ch'io sia atto a rispondervi. ${ }^{23}$

Il dono diventa poi il pretesto per l'ennesima tirata antipedantesca, che un Aretino fiero di portare il vessillo dell'«ignoranza in su la palma de la mano» proclama con l'assertività aggressiva e sboccata che caratterizza buona parte delle sue prese di posizione in materia di poetica:

Veramente io, che tanto andai a la scuola, quanto intesi la «Santa croce, fatimi bene imparare», componendo ladramente merito scusa, e non quegli che lambicano l'arte de i Greci e de i Latini, tassando ogni punto e imputando a ogni «che», facendosi riputazione con l'avertenza de l'acuto d'una vocale. «Io (disse Gian Giordano) non so né ballar, né cantare, ma chiavarei come un asenazzo». Sì che leggendo le mie coglionerie scusatimi con voi stesso per ch'io son più tosto Profeta che Poeta. ${ }^{24}$

Tanto che, in conclusione, l'excusatio e la topica professione di umiltà banno un sapore ambiguo, non privo di una punta di veleno. Perché 
l'Aretino-profeta che richiama $i$ fasti del vitiorum ac virtutum demonstrator, da un lato, riconosce coram populo a Dolce il grado di poeta, ma, dall'altro, prende le distanze dall'universo poetico in cui lo ha appena collocato rivendicando alla propria musa una concretezza espressiva ben diversa. La stessa sensazione si ricava dalla lettura del sonetto al seguito della missiva:

Dolce, Ambrosia d'Apollo, le cui stille Spruzzon liquor di gloria e d'intelletto, Tal desio de i miei scritti ardevi il petto, Che n'abbiate a scoprir tante faville?<smiles>[AlH2]</smiles>

Io me conosco, e voi; e so che l'arte

Vostra è del dire, e so che chiaro sete

In quegli onor che ponno dar le carte.

Alla lode delle virtù di un Dolce desideroso di essere eternato negli scritti del maestro, ${ }^{25}$ corrisponde, nella prima terzina, l'esibizione di una sapienza da parte dell'io aretiniano da cui deriva una chiara coscienza dei propri limiti e della propria radicale alterità. Come a voler dare atto al compare di un primato poetico, limitandolo però al mondo di carta dei verba e della retorica e riservando implicitamente a sé la sfera prediletta delle res. In questo senso, per inciso, andranno decrittate anche le nuove professioni di umiltà contenute nell' importante lettera del I9 gingno I539, la III del secondo libro, con la quale Dolce, di nuovo coinvolto in una triangolazione tra Aretino e Bembo, riceve un sonetto celebrativo per la fresca nomina del cardinale. ${ }^{26}$

Il cerchio si chiude con la responsiva senza data di Dolce che viene pubblicata come XL della giunta al primo delle Lettere del I542. La manifestazione di gratitudine per l'inclusione nel novero degli illustri destinatari dell'epistolario aretiniano si accompagna a una riflessione sull'immortalità offerta dal maestro al discepolo e sulla necessaria gratuità del dono che riecheggia quella proposta nell'epistola XLII:

M'è dunque cara la lettra e 'l Sonetto a me indrizzato e posto in compagna de le lettre scritte a tanti Principi e uomini che meritano grandemente. M'è cara dico ne la guisa che debbono esser care quelle cose che danno la immortalità [...]. Tutto che voi non aspettiate pagamento alcuno da me, perché il dono non sarebbe dono. ${ }^{27}$ 
Per arricchire il quadro delle intersezioni tra generi lirico e epistolare nel percorso biografico e letterario di Dolce, sarà utile allargare lo sguardo alla corrispondenza con Varchi. Un interlocutore privilegiato in materia poetica col quale si instaura un dialogo alla pari tra quasi coetanei, essenziale per più rispetti in vari contesti, non ultimo quello dello scontro con Ruscelli. ${ }^{28}$ La circolazione a mezzo epistolare di frammenti lirici e di riflessioni tecniche sui medesimi si conferma uno dei collanti che contribuiscono a vincolare un intero universo di relazioni sociali, come quello che fa capo al consesso accademico degli Infiammati di Padova. Lo vediamo con chiarezza nella lettera a Varchi del 29 dicembre I540, dove Dolce chiede con ansia di essere messo a parte di un sonetto di Bembo alla cui lectura, suo malgrado, non ba potuto assistere:

Monsignor Gradinico [...] mi raccontò lei questi giorni a dietro aver letto nell'Academia quel sonetto del vostro et mio reverendissimo signor cardinale che incomincia "Se la più dura quercia che l'Alpe haggia" [...]. Onde, poscia che io non posso essere a parte del comune diletto et utile di molti in porger soave cibo alle orecchie et al mio piccolo intelletto delle dotte et diverse lettioni che io odo dal sermone di più huomini letterati vostra signoria legger quasi di continovo, presa securtà nella molta sua humanità et cortesia, da lei verso di me assai prima che hora dimostrata, di pregarla che le sia in grado di farmi goder partecipe di detti versi, acciò che io possa in parte prender frutto, assente, di quello che non m'è concesso di poter presente. ${ }^{29}$

In cambio della condivisione a distanza del rito accademico, resa possibile solo attraverso $i$ canali della corrispondenza, Dolce offre al sodale $i$ propri servigi in Laguna, ringraziandolo nella chiusa di aver dato seguito con una risposta per le rime a un suo sonetto:

Et perché fra tanto io penso che vostra signoria mi tenga nella sua buona gratia, altro non le dirò se non che ella si degni di disponer de le picole forze mie con quella sicurtà con che ella può disponer del più caro et affettionato amico ch'ella habbia [...]. Hebbi molti dì sono la bella et dotta risposta al sonetto che io le mandai, et la ringratio senza numero di volte. ${ }^{30}$

Non si tratta in realtà di un sonetto qualunque, ma, con ogni probabilità, del componimento a sua volta oggetto della lettura accademica di Varchi che aveva consacrato la cooptazione di Dolce tra gli 
Infiammati. ${ }^{3 \mathrm{I}}$ L'episodio è documentato dalla lettera senza data riconducibile all'ottobre I540 in cui il letterato fiorentino descrive lo scambio delle rime annunciandone la prossima declamazione di fronte all'illustre platea padovana:

Ho ricevuto un molto dolce, leggiadro e amorevole sonetto di vostra signoria, nel quale ho riconosciuto l'ingegno, l'umanità e cortesia sua e sopra tutto il troppo amore ch'ella mi porta, avendo detto nel primo quaternario quello ch'io, non solo non riconosco in me, né chieggio, ma né ancora osarei di desiderarlo. Io, signor, ho fatto la risposta come ho saputo e potuto, non come arei voluto e come devea. Vostra signoria mi scusi, per ciò che [...] io sono stato sforzato a pigliare il carico di leggere publicamente l'Etica nella nostra Academia, nella quale, domenica che viene, recitarò secondo l'usanza il vostro sonetto e la risposta mia. ${ }^{32}$

Secondo un'ipotesi persuasiva avanzata a partire dall'osservazione di Varchi sulla prima quartina del sonetto che Dolce gli ha dedicato, il riferimento sarebbe ai componimenti Varchi, mentre che voi spiegando l'ali (proposta) e Dolce, le prose mie, né i versi tali (risposta). ${ }^{33}$ Ma ciò che qui preme osservare è la conferma del rapporto strettissimo, quasi simbiotico, tra frammento lirico e lettera, in virtù del quale il gioco dialogico delle rime di corrispondenza si completa collimando in un commento formulato con l'agio della prosa su sponda epistolare: dalla semplice espressione di gratitudine alla riflessione mirata sulla sostanza dei testi.

C'è poi una splendida lettera che svela come poche altre l'intreccio di relazioni interpersonali sotteso all'impressionante traffico epistolare di materiale poetico. Da Padova, il is ottobre 1536, Varchi ringrazia Dolce per un sonetto promettendone uno in cambio e condividendo il progetto di una prossima visita a Venezia nel corso della quale prevede di far visita all'Aretino:

le dirò solo che quando egli [scil. Antonio, familiare di Varchi] mi portò il leggiadro e dotto sonetto di vostra signoria io ne faceva appunto uno indiritto a lei, il quale sarà con questa, ancor che indegno di lei, del che quella non solo m'iscuserà e perdonerà, ma l'accetterà in quella parte $\mathrm{e}$ con quel buon animo ch'io gliele mando. [...] fra tre giorni o quattro al più lungo verrò costì per far parte del mio debito con vostra signoria $\mathrm{e}$ rivedere il nostro signor messer Pietro divino, al quale vostra signoria si degnerà raccomandarmi infinite volte. ${ }^{34}$ 
Ancora una volta la poesia si dimostra zona franca, polo di aggregazione privilegiato tra due letterati come Bembo e Aretino, figure in verità molto meno lontane di quanto talora ci si ostini a pensare. ${ }^{35}$ Attorno alle Stanze per Angela Serena, pronubi Varchi e Dolce, il futuro cardinale e il Flagello de' Principi si incontrano e dialogano per interposta persona:

Monsignor Bembo a vostra signoria e a messer Pietro si raccomanda, le stanze del quale gli son piaciute a maraviglia e le rimanda con alcuni suoi avertimenti, ma di tutto parleremo a bocca in breve, come ho detto di sopra, che mercoledì o giovedì sarò in casa detto messer Pietro. In questo mentre, vedrà di fare la risposta al sonetto come potrà il meglio. ${ }^{36}$

Nel finale c'è ancora spazio per introdurre a Dolce un aspirante poeta che gli manderà uno scambio di sonetti a mo' di biglietto da visita e per raccomandarsi all'Aretino, pregustando un incontro in cui la fruizione dei testi potrà essere finalmente de visu e non più mediata dal mezzo epistolare:

due dì sono me ne fu mandato un [scil. sonetto] da Firenze da un giovanetto, che per non avere altro lo manderà a vostra signoria con la risposta fatta subito, come per se stessa vedrà vostra signoria, alla quale di continovo mi offero e raccomando, pregandola si degni raccomandarmi di nuovo a messer Pietro e dirgli che ci vengo solo per vedere e visitar voi duoi e leggere compositioni di tutti due. ${ }^{37}$

Lo spoglio di ciò che resta del prezioso carteggio con Varchi porta persino a imbattersi in esercizi di esegesi condotti sul testo lirico. Esemplare al riguardo la lettera a Dolce del 20 febbraio I54I, ricchissima di spunti per sondare il livello del confronto culturale a tutto campo in atto fra i due. Al via vai di libri sull'asse Padova-Venezi $a^{38}$ corrisponde il solito movimento di sonetti: Varchi comunica di averne appena composto uno per Alessandro Piccolomini e promette di recapitarlo anche a Dolce una volta consegnato al destinatario. Da qui sipassa a discutere l'interpretazione di un luogo del sommo modello petrarchesco (Qual ventura mi $\mathrm{fu}$, quando da l'uno, Rvf 233), materia viva al punto da poter essere accostata senza soluzione di continuità alla riflessione sulla nuova lirica contemporanea, direttamente germinata da quel corpo antico:

Io ho fatto stanotte il sonetto al Piccolomini, ma ancora non l'ho scritto e ora non ho tempo; come l'avrò dato a lui, lo mandarò a vostra signoria 
[...]. Quanto al sonetto, "Qual ventura mi fu", io non ho veduto cosa niuna, ma per quello che mi pare così a mente, egli intende, come si dice volgarmente, che il male de gli occhi si appicca e che avendo guardato madonna Laura, ch'avea male al destro occhio, venne male ancora a lui e se gli appiccò. ${ }^{39}$

Il saggio di lectura, sollecitato con ogni evidenza da un quesito proposto da Dolce in una precedente missiva oggi perduta, prosegue sino al termine della lettera, dove Varchi si dichiara interessato a raccogliere nuove opinioni in seno a un dibattito che rimane per sua natura aperto ad infinitum:

Ho dette queste cose così all'improviso e in aria non perché non creda che vostra signoria non le sappia molto meglio di me, ma per sodisfarle in tutto quello mi ricerca, come farò sempre. Vostra Signoria sarà contenta avisarmi se c'ha spositione alcuna nuova, non detta da gli altri, che la mia penso che sarà commune e rozza. ${ }^{40}$

Se, infine, l'esegesi dell'ipotesto petrarchesco si fa rito sociale da condividere nel milieu accademico cosi come nella corrispondenza privata, lo stesso spazio epistolare può diventare il laboratorio ideale per perfezionare a quattro mani la stesura di un prodotto poetico che nello sdoppiamento della figura autoriale trova forse una delle sue peculiarità filologiche più rilevanti. E quanto si scorge tra le righe della lettera del I7 ottobre I539, nella quale Varchi si spinge a richiedere uno spassionato expertise di Dolce sulle qualità di un giovane poeta fiorentino:

Io mandai a vostra signoria il sonetto amendato, ma avendo quella risposto sì tosto e sì dottamente e con tanta leggiadria, io lascierò star come prima; rimandole l'altro un poco ritocco e uno di quel Martello, che è un giovane nobile e molto letterato, greco e latino di suo tempo, e di nuovo si è dato al toscano: arei caro intender per lettere di vostra signoria quello gli pare, che me, oltra l'altre cose, potrebbe ingannare la patria e l'amicizia. Honne avuto oggi uno altro, che anche quello gli vo' mandare. ${ }^{4 \mathrm{I}}$

La conferma del fatto che alla fine degli anni' 30 Dolce può già vantare una confidenza col genere lirico che, nel corso del decennio successivo, indurrà Giolito a coinvolgerlo coi gradi di autore e curatore nel grande cantiere delle antologie. 
I. Lodovico Dolce, Lettere, a cura di P. Procaccioli, Manziana, Vecchiarelli, 20 i 5 , lett. 62, p. 179. Da questa silloge sono ricavate anche tutte le citazioni dalle lettere a Dolce, escluse quelle di Pietro Aretino.

2. Dolce, Lettere, p. i 80.

3. Il sonetto Al poco lume e molto spazio d'ombra si legge ora nell'appendice di Rime diverse di molti eccellentissimi autori (Giolito I545), a cura di F. Tomasi e P. Zaja, Torino, RES, 200I, p. 369. Della figura di Crivelli si è occupato M. Firpo, Artisti, gioiellieri, eretici. Il mondo di Lorenzo Lotto tra Riforma e Controriforma, Roma-Bari, Laterza, 200I, pp. 21 2-20.

4. Dolce, Lettere, lett. 63, p. I 82.

5. F. Tomasi, Le ragioni del "moderno" nella lirica del XVI secolo tra teoria e prassi (2008), in Studi sulla lirica rinascimentale (I540-I570), Roma-Padova, Antenore, 20 I 2, Pp. 3-24, a p. Is.

6. Il fenomeno è già approfondito dallo stesso LoDovico Dolce, I quattro libri delle Osservationi, a cura di P. Guidotti, Pescara, Libreria dell'Università, 2004, pp. 500-505. Sul valore della «poesia come forma di comunicazione sociale, con una estesa gamma di funzioni», letto proprio alla luce dell'alta frequenza dei testi di corrispondenza si è soffermato A. Quondam, Dall'abstinendum verbis alla "locu₹ione artificiosa". Il petrarchismo come sistema linguistico della ripetizione (1973), in Il naso di Laura. Lingua e poesia lirica nella tradizione del classicismo, Modena, Panini, I 99 I, pp. I 8 I-99, a p. I 88. Sullo specifico è ora d'obbligo il rinvio ai fondamentali contributi di F. Tomasi, «I più vaghi e i più soavi fiori». Alcuni aspetti delle antologie liriche del secondo Cinquecento (200I), in Studi sulla lirica rinascimentale, cit., pp. 25-94, alle pp. 56-58, e di P. Zaja, Intorno alle antologie. Testi e paratesti in alcune raccolte di lirica cinquecentesche, in «I più vaghi e i più soavi fiori». Studi sulle antologie di lirica del Cinquecento, a cura di M. Bianco e E. Strada, Alessandria, Edizioni dell'Orso, 200 I, pp. I I 3-45, alle pp. I 26-28.

7. Per un primo orientamento su consistenza e temi del corpus lirico dolciano mi permetto di rinviare a P. Marini, Per l'edizione commentata della lirica di Lodovico Dolce, in I cantieri dell'italianistica. Ricerca, didattica e organizzazione agli inizi del XXI secolo, Atti del XVII congresso dell'ADI (Roma Sapienza, i 8-2 I settembre 20I 3), a cura di B. Alfonzetti, G. Baldassarri e F. Tomasi, Roma, Adi editore, 2014, pp. I-8 [pubblicazione elettronica disponibile in formato pdf sul sito web di libero accesso http://www.italianisti.it/]; P. Marini, «Non per human lavoro». Note su Dolce lirico e la questione dei sonetti spirituali, in Per Lodovico Dolce. Miscellanea di studi, I, Passioni e competenze del letterato, a cura di P. Marini e P. Procaccioli, Manziana, Vecchiarelli, 2016, pp. 41-89.

8. Pietro Aretino, Lettere. Libro I, a cura di P. Procaccioli, Roma, Salerno Editrice, I997, lett. 77 , p. I 37 .

9. Aretino, Lettere. Libro I, lett. i 28, p. I97. Lo scambio di sonetti tra Dolce e la Gambara è menzionato da G. Cingolani, Su uno scambio di sonetti fra Giulio Camillo e Veronica Gambara, in Il petrarchismo. Un modello di poesia per l'Europa, a cura di F. Calitti e R. Gigliucci, Roma, Bulzoni, 2006, vol. II, pp. 349-64, a p. 354. 
Iо. DoLce, Lettere, lett. 57, p. I70.

i i. Dolce, Lettere, lett. 56, pp. i68-69.

I 2. Aretino, Lettere. Libro I, lett. 295, p. 406.

I 3. Dolce, Lettere, lett. 2, p. 47.

i4. Pietro Aretino, Lettere. Libro II, a cura di P. Procaccioli, Roma, Salerno Editrice, I998, lett. 289, p. 3 I 8 . Si è giustamente osservato che «è da affermazioni come questa [...], e da lettere come la presente, che nasceva l'immagine pubblica di Dolce come curatore» (Dolce, Lettere, lett. 73, p. 207, n. 107). Il coinvolgimento di Dolce nell'allestimento delle Lettere è stato indagato nel dettaglio dallo stesso P. Procaccioli, Cosi lavoravano per Aretino. Franco, Dolce e la correzione di Lettere, $I$, in «Filologia e Critica», XXI (I996), pp. 264-80.

i 5 . Dolce, Lettere, lett. 3, pp. 49-5 I.

i6. Aretino, Lettere. Libro I, lett. i 55, pp. 229-32.

I7. Dolce, Lettere, lett. 3, p. 50.

I 8. Dolce, Lettere, lett. 3, p. 49.

i 9. Dolce, Lettere, lett. 3, pp. 49-50.

20. Senza risalire ai precedenti dell'Antologia Palatina e della lirica trobadorica, basterà ricordare Giacomo da Lentini, Meravigliosa-mente, 7-9: «così, bella, facc'eo / che 'nfra lo core meo / porto la tua figura». Nel modello petrarchesco, oltre a $R v f$ 96, interessa qui segnalare fra i vari passi esemplari i vv. 9-I I del sonetto $R v f$ I 30 («Et sol ad una imagine m'attegno, / che fe' non Zeusi, o Prasitele, o Fidia, / ma miglior mastro, et di piú alto ingegno») per l'accostamento tra l'opera di Amore e degli artisti sommi. Un tema che torna in Tebaldeo (son. 5 56, I-6: «Qual Zeusi, Apelle, o qual pictor più degno, / sì ben ritratte mai de uno huom figura? / Qual Prasitel mai finse in petra dura / imagine più vera col suo ingegno? / Come Amor dentro al pecto il bel disegno / m'impresse de costei») e, in forma diversa, in Bembo (son. I 9, I-6: «O imagine mia celeste e pura, / che splendi più che 'l sole agli occhi miei / e mi rassembri 'l volto di colei, / che scolpita ho nel cor con maggior cura, / credo che 'l mio Bellin con la figura / t'abbia dato il costume anco di lei»). Un approfondimento della questione, che coinvolge anche il tema del ritratto in poesia, in E. Jager, The book of the heart, Chicago-London, University of Chicago Press, 2000, pp. 65-86; L. Bolzoni, Il cuore di cristallo. Ragionamenti d'amore, poesia e ritratto nel Rinascimento, Torino, Einaudi, 2010, pp. 308-25; F. Pich, I poeti davanti al ritratto. Da Petrarca a Marino, Lucca, Maria Pacini Fazzi, 2010, pp. 3 I-43.

2 I. Dolce, Lettere, lett. 3, pp. 50-5 I.

22. Si tratta della ben nota autoproclamazione di Aretino scriba Naturae, di cui qui è appena il caso di riportare alcuni stralci tra i più memorabili: «Andate pur per le vie che al vostro studio mostra la natura, se volete che gli scritti vostri faccino 
stupire le carte dove son notati [...]. E per dirvelo, il Petrarca e il Boccaccio sono imitati da chi esprime i concetti suoi con la dolcezza e con la leggiadria con cui dolcemente e leggiadramente essi andarono esprimendo i loro, e non da chi gli saccheggia non pur de i "quinci”, de i “quindi”, e de i "soventi”, e de gli “snelli", ma de i versi interi [...]. O turba errante, io ti dico e ridico che la poesia è un ghiribizzo de la natura ne le sue allegrezze, il qual si sta nel furor proprio, e mancandone il cantar Poetico diventa un cimbalo senza sonagli, e un campanil senza campane [...]. La natura istessa, de la cui semplicità son secretario, mi detta ciò che io compongo [...]. Sì che attendete a esser scultor di sensi, e non miniator di vocaboli», Aretino, Lettere. Libro I, lett. i 55, pp. 229-32.

23. Aretino, Lettere. Libro I, lett. 249, p. 346. I sonetti di Dolce qui menzionati, come molti altri componimenti cui si allude nella sua corrispondenza, risultano irreperibili. Quella dell'estrema dispersione del materiale poetico è proprio una delle informazioni più rilevanti - e, aggiungerei, più sconfortanti in prospettiva ecdotica - che si ricava dallo spoglio delle lettere di e a Dolce.

24. Aretino, Lettere. Libro I, lett. 249, p. 347. Da notare che il livello culturale dell'alfabetizzazione di base rappresentato dal manuale della Santacroce e qui provocatoriamente esibito da Aretino è il medesimo indicato dalla ruffiana Gemma nel progetto didattico di scuola per fanciulle proposto nell'Ipocrito (II i 6 I). Sullo specifico rinvio a P. Marini, «Più pro fa il pane asciuto in casa sua...». Formule proverbiali e sentenziose in Pietro Aretino, in Il proverbio nella letteratura italiana dal XV al XVII secolo, Atti delle Giornate di studio, Università di Roma Tre-Fondazione Marco Besso (Roma, 5-6 dicembre 201 2), a cura di G. Crimi e F. Pignatti, Manziana, Vecchiarelli, 20I4, pp. 67-I I I, alle pp. 88-89.

25. È il desiderio che, come si è appena visto, Dolce aveva espresso anche nella lettera del I 8 giugno i 537 .

26. Aretino, Lettere. Libro II, lett. i i i, p. i i 9: «Eccovi il Sonetto che a la insufficienza mia ha fatto comporre non la gara, ma l'affezzione ch'io porto al Bembo [...]. Egli è certo che io non mi son mosso a farlo avenga che lo stuolo di tutti i Poeti onori co i versi il dono che del Cappello gli ha meritissimamente fatto Paolo III, né per darmi ad intendere d'essere atto di por lo stile in sì nobil materia, ma perché ne le nozze de i padroni ci cantano fino a i servi. Or se nel leggerlo non ci trovate quello spirito che si richiede a un tanto uomo, escusatimi con dire che a scrivere di lui bisognaria o la sua penna istessa, o calamo simile al vostro».

27. Dolce, Lettere, lett. 34, pp. I 2 I-22.

28. Cfr. Dolce, Lettere, lett. 31-32, pp. I08-i 3; lett. I I 3, pp. 28 I-88.

29. Dolce, Lettere, lett. 7, pp. 57-58.

30. Dolce, Lettere, p. 58.

3r. Documentano l'ingresso di Dolce nell'Accademia, da ricondurre ai primi mesi del i 540, le lettere a Varchi dell'8 e del 20 gennaio (DoLCE, Lettere, lett. 4-5, pp. 52-54). Sulla «solerte attività didattica» dispiegata da Varchi nel programma di 
letture proposte agli Infiammati si sofferma V. Vianello, Il letterato, l'Accademia, il libro. Contributi sulla cultura veneta del Cinquecento, Padova, Antenore, i988, p. 75. Prezioso al riguardo anche l'intervento di F. Tomasi, Le letture di poesia e il petrarchismo nell'Accademia degli Infiammati, in Il petrarchismo. Un modello di poesia per l'Europa, cit., pp. 229-50.

32. Dolce, Lettere, lett. 70, p. $20 \mathrm{I}$.

33. Cfr. Dolce, Lettere, lett. 70, p. 20 I, n. 90. Dei 24 casi di rime di corrispondenza sin qui censiti, a testimoniare la vitalità del dialogo poetico fra il nostro e i letterati suoi contemporanei, ben 4 sono relativi a botta e risposta con Varchi: La bella donna, che tra Bice e Laura (Varchi), Quei che cantò molti anni e pianse Laura (Dolce); Varchi, che lieto al glorioso monte, Cosi vosco il mio nome altero monte; Varchi, che i lieti e bei vicini campi, Dolce se gl'amorosi ardenti vampi; Varchi, mentre che voi spiegando l'ali, Dolce, le prose mie, né i versi tali: i testi si leggono in Benedetto Varchi, Opere, ora per la prima volta raccolte, Trieste, Lloyd Austriaco, I 859, II, pp. 924-25, 965-66.

34. Dolce, Lettere, lett. 55, p. I66.

35. I termini effettivi del loro complesso rapporto sono precisati in P. Procaccioli, Due re in Parnaso. Aretino e Bembo nella Venezia del doge Gritti, in Sylva. Studi in onore di Nino Borsellino, a cura di G. Patrizi, Roma, Bulzoni, 2002, I, pp. 207-3 I.

36. Dolce, Lettere, lett. 55, pp. I66-67. Delle reciproche richieste di sonetti tra Bembo e Aretino in cui Dolce è coinvolto direttamente come mediatore resta traccia nelle lett. 2 e io (Dolce, Lettere, pp. 47-48, 63).

37. Dolce, Lettere, lett. 55, p. I67.

38. Dolce, Lettere, lett. 72, pp. 203-204: «mi mandi quanto prima sì le cose ch'io per l'altra le chiesi e sì di più un Vergilio di quelli coll'indice e una Poetica di quelle di messer Bernardino Daniello [...]; e così le rime del Brocardo, dove sono quelle del Molza [...]. Quanto al Dialogo di messer Sprone vostra signoria lo tenga quanto le torna bene, un anno o due non importano».

39. Dolce, Lettere, lett. 72, p. 204.

40. Dolce, Lettere, lett. 72, p. 205.

4I. Dolce, Lettere, lett. i i 2, p. 280. 